\title{
Driving the urge to eat
}

\section{Cf \\ the effects of cannabinoids on feeding may be due to a selective increase in $\beta$-endorphin release}

Exogenous cannabinoids promote feeding in sated animals, which is thought to involve a neural circuit in the hypothalamus, but the underlying details have not been established. Now, Koch et al. show that cannabinoid receptor 1 (CB1R)selective agonists increase feeding in mice by activation of hypothalamic pro-opiomelanocortin-expressing neurons (POMC neurons).

The hypothalamus has a central role in the regulation of food intake. Typically, activation of POMC neurons in the arcuate nucleus of the hypothalamus promotes satiety.

Interestingly, however, the authors found that systemic doses of CB1R agonists that increased feeding in mice also increased cFOS expression, a marker of neuronal activation, in POMC neurons. Moreover, in mice expressing inhibitory DREADD (designer receptors exclusively activated by designer drugs) in POMC neurons, administration of clozapine$\mathrm{N}$-oxide (CNO) to activate the inhibitory DREADD reduced CB1R agonist-induced feeding. Together, these data suggest that activation of POMC neurons drives the feeding response to cannabinoids.

POMC neurons release two peptides, both encoded by the Pomc gene: a-melanocyte-stimulating-hormone ( $a-\mathrm{MSH})$, which is usually considered to be an appetite-suppressing peptide, and $\beta$-endorphin, which can increase appetite. The protein levels, but not the transcript levels, of the proprotein convertase 1 (PC1) and PC2, which are involved the production of $\alpha-\mathrm{MSH}$ and $\beta$-endorphin, were increased in the hypothalamus in response to CB1R agonist administration. Furthermore, CB1R agonists increased $\beta$-endorphin secretion in the paraventricular nucleus (PVN), a region of the hypothalamus that POMC neurons project to and that is implicated in feeding behaviour, but they did not affect $\alpha$-MSH secretion. Thus, the effects of cannabinoids on feeding may be due to a selective increase in $\beta$-endorphin release.

In support of this possibility the administration of naloxone, an antagonist of the $\mu$-opioid receptor (which binds $\beta$-endorphin), to the PVN prevented increased feeding in response to $\mathrm{CB} 1 \mathrm{R}$ agonists.

The authors next investigated how cannabinoids might modulate the production and secretion of peptides by POMC neurons. They showed that CB1Rs are found not only on the presynaptic terminals of POMC neurons but also intracellularly in mitochondria. Moreover, they showed that CB1R agonists induce a number of changes in mitochondrial dynamics in these neurons, including increased mitochondrial respiration, which was associated with increased reactive oxygen species levels. Previous studies had shown that mitochondrial adaptations can occur in POMC neurons in response to factors such as feeding state and may contribute to regulation of food intake.

The administration of CB1R agonists increased the hypothalamic levels of mitochondrial uncoupling protein 2 (UCP2), which can be induced in response to increased reactive oxygen species levels, and had previously been linked to hypothalamic modulation of feeding behaviour. Strikingly, mice lacking Ucp2 failed to show increased feeding, changes in PC1 and PC2 levels, or increased $\beta$-endorphin release in the PVN in response to $\mathrm{CB} 1 \mathrm{R}$ agonists. Thus, UCP2 is an essential mediator of the cannabinoid-induced feeding response, and it provides a molecular link between CB1R-mediated modulation of mitochondrial dynamics in POMC neurons and increased $\beta$-endorphin secretion.

Together, these findings suggest that, in addition to their established role in the suppression of feeding, POMC neurons can also drive increased food intake, at least in response to exogenous cannabinoids. They also reveal an intracellular mechanism by which CB1R agonists promote feeding, which could prove useful for the therapeutic modulation of appetite.

Fiona Carr

ORIGINAL RESEARCH PAPER Koch, M. et al. Hypothalamic POMC neurons promote cannabinoid-induced feeding. Nature http://dx. doi.org/10.1038/nature14260 (2015) 